\title{
FLUTTER SUPPRESSION DIGITAL CONTROL LAW DESIGN AND TESTING FOR THE AFW WIND-TUNNEL MODEL
}

\section{Vivek Mukhopadhyay}

July 1992

$$
\begin{aligned}
& \text { (NASA-TM-107652) FLUTTER } \\
& \text { SUPPRESSION DIGITAL CONTROL LAW } \\
& \text { UESIGN AND TESTING FOR THE AFW } \\
& \text { WIND-TUNNEL MOOEL (NASA) } 8 \mathrm{p}
\end{aligned}
$$

N92-31350

Unclas

$63 / 05 \quad 0115455$

\section{N/Sก


5 


\title{
FLUTTER SUPPRESSION DIGITAL CONTROL LAW DESIGN AND TESTING FOR THE AFW WIND-TUNNEL MODEL
}

\author{
Vivek Mukhopadhyay * \\ NASA Langley Research Center, Hampton, Virginia 23665
}

\section{Abstract}

Design of a control law for simultaneously suppressing the symmetric and antisymmetric flutter modes of a sting mounted fixed-in-roll aeroelastic wind-tunnel model is described. The flutter suppression control law was designed using linear quadratic Gaussian theory, and involved control law order reduction, a gain root-locus study and use of previous experimental results. A $23 \%$ increase in the open-loop flutter dynamic pressure was demonstrated during the wind-tunnel test. Rapid roll maneuvers at $11 \%$ above the symmetric flutter boundary were also performed when the model was in a free-toroll configuration.

\section{Nomenclature}

A, B control law state-space matrices

C, D control law output matrices

$\mathrm{B}_{\mathrm{O}} \quad$ Kalman state estimator gain matrix

$\mathrm{C}_{0}$ optimal regulator gain matrix

E expectation operator

F, G plant state-space matrices

$\mathrm{G}_{w} \quad$ gust input matrix

g gravitational acceleration constant

H sensor output matrix

I identity matrix

M Mach number

$\mathrm{P}$ estimator Riccati equation solution

q dynamic pressure, psf

qf flutter dynamic pressure, psf

Q1 plant output weighting matrix

$\mathrm{Q}_{2}$ control input weighting matrix

$R_{v}$ measurement noise intensity matrix

$\mathbf{R}_{\mathbf{w}} \quad$ gust input noise intensity

S regulator Riccati equation solution

s Laplace variable

T sample period, seconds

$t$ time, seconds

u control input vector

$\mathrm{v}$ measurement noise vector

w gust input noise

$\omega$ frequency, radians/second

$x$ plant state vector

$\mathrm{x}_{\mathrm{c}} \quad$ control law state vector

y measurement vector

z $\quad$ accelerometer output, g's

$\delta \quad$ control surface angular position, degrees

Subscripts:

LEI wing leading edge inboard

LEO wing leading edge outboard

TEI wing trailing edge inboard

TEO wing trailing edge outboard

tip wing tip

Abbreviations:

AFW active flexible wing

$C L$ closed loop

CPE controller performance evaluation

FSS flutter suppression system

LQG linear quadratic Gaussian

$O L$ open loop

psf pounds per square foot

RMLA rolling maneuver load alleviation

rms root mean square

SISO single-input single-output

\section{Introduction}

A summary of the Active Flexible Wing (AFW) Program is presented in Ref. 1. Within the operating range of the Langley Research Center Transonic Dynamics Tunnel, the sting mounted AFW aeroelastic model had both symmetric and antisymmetric flutter modes, in a fixed-in-roll configuration, and a symmetric flutter mode only, when the model was in a free-to-rol configuration. The active flutter suppression system (FSS) test goals were to demonstrate: a) simultaneous symmetric and antisymmetric flutter suppression for the fixed-in-roll configuration, and b) symmetric flutter suppression in the freeto-roll configuration. An additional goal was to test a rolling maneuver load alleviation system along with the FSS above the open-loop flutter boundary. Since the free-to-roll symmetric flutter and the fixed-in-roll symmetric and antisymmetric flutter modes had very similar characteristics, a single FSS control law was designed and demonstrated for both the flutter test configurations, a) and b) as stated above. This paper addresses the mathematical modeling, control law design and wind-tunnel test results.

\section{AFW Equations of Motion}

The description of the AFW aeroelastic wind-tunnel model and the wing-tip ballast stores, including details of the accelerometer sensor positions and multiple control surface actuation capabilities are provided in Ref. 2 . The accelerometer sensors and the control surface locations on the wing-plan form are shown in figure 1 . The development of the aeroelastic equations of motion is described in Ref. 3. The equations for the symmetric and antisymmetric motion were developed separately, using ten flexible modes for each configuration. The flexible mode shapes and natural frequencies were derived from a finiteelement modal analysis and were corrected using ground vibration test data.

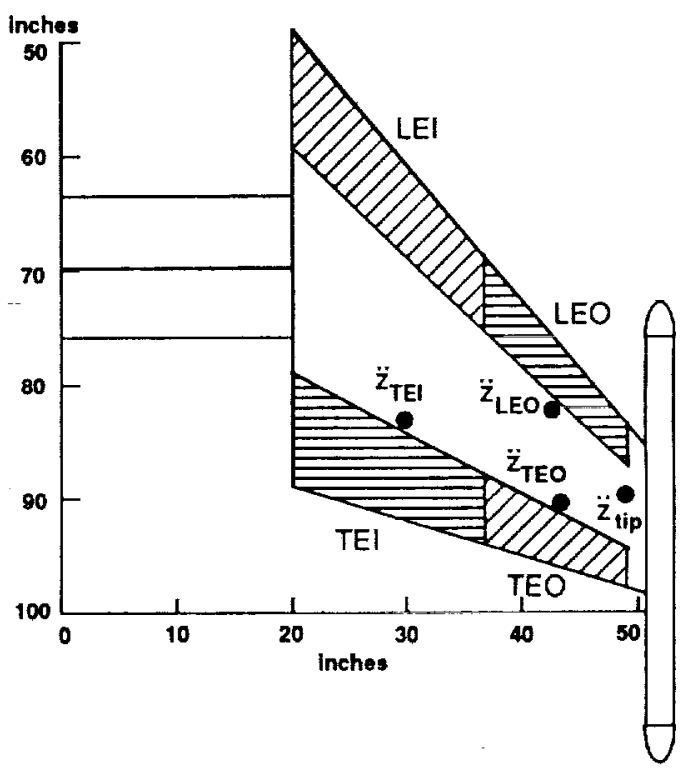

Figure 1. Accelerometer and control surface locations on AFW wing plan form.

*Associate Fellow, ALAA 
State-space equations: A set of state-space mathematical models were developed for control law design ${ }^{3}$. For the aeroelastic equations, the doublet-lattice oscillatory aerodynamics approximation used four aerodynamic lag terms for each flexible mode. In addition, the state-space models included corrections for control surface effectiveness based on results from the 1989 wind-tunnel test ${ }^{2}$, and the third-order transfer functions of the actuator dynamics derived from ground test of the unloaded control surfaces. A Dryden gust spectrum transfer function, driven by a white noise process was used to simulate the random vertical gust of the wind-tunnel. The complete linear equations of motion at a specified dynamic pressure were expressed by the state-space equations,

and

$$
\begin{aligned}
\mathrm{dx} / \mathrm{d} t & =\mathrm{Fx}+\mathrm{Gu}+\mathrm{G}_{\mathrm{w}} \mathrm{w} \\
\mathrm{y} & =\mathrm{Hx}+\mathrm{v}
\end{aligned}
$$

where $\mathrm{x}$ is the state vector, $\mathrm{u}$ is the control input vector, $\mathrm{w}$ is the gust input noise, $y$ is the accelerometer sensor output vector, and $\mathrm{v}$ is the measurement noise vector. Equations (1) and (2) were scaled such that the units of the control inputs were in degrees, the units of the sensor outputs were in g's, and the gust input units were in feet/second.

Open-loop dynamic pressure root-locus: Using these state-space mathematical models at six dynamic pressures, $q=100,150$, $200,250,300$ and 350 psf, the flexible-mode root-loci with dynamic pressure were studied. The open-loop, dynamic pressure root-locus of the first four flexible symmetric and antisymmetric modes, for the fixed-in-roll configuration, are shown in figures 2 and 3 , respectively. The figures 2 and 3 indicate that the second and third flexible mode frequencies coalesced to produce the flutter instability. The unstable mode was primarily wing-tip torsion, for both the symmetric and the antisymmetric motions. The sixth and seventh symmetric flexible mode frequencies also tended to coalesce (not shown in figure 2). At Mach 0.5 , the analytical open-loop symmetric flutter dynamic pressure was estimated to be $248 \mathrm{psf}$ at $11.2 \mathrm{~Hz}$. The analytical open-loop antisymmetric flutter dynamic pressure was estimated to be 233 psf at $10.9 \mathrm{~Hz}$. The closed-loop dynamic-pressure root-locus is also shown in figures 2 and 3 and will be discussed later.

\section{Control Law Design}

The flutter suppression design objective was to develop loworder robust digital control laws which would simultaneously suppress the symmetric and antisymmetric flutter modes of the model in the fixed-in-roll configuration with allowable control surface activity. The maximum permissible control surface rms deflection and rates were 1.0 degree (at $11.2 \mathrm{~Hz}$ flutter frequency) and 75 degrees/second, respectively. From the 1989 test $^{2}$, the antisymmetric flutter frequency was known to be 1.8 $\mathrm{Hz}$ below the theoretical value. The control law was also required to be sufficiently robust to compensate for this difference.

The FSS control laws were designed using linear quadratic Gaussian (LQG) theory and involved control law order reduction, a gain root-locus study, and use of previous experimental results ${ }^{2}$. Since the symmetric and antisymmetric flutter modes had very similar characteristics for the fixed-in-roll configuration (see figures 2 and 3), a single FSS control law was designed to suppress both the flutter modes. This control law used the $\ddot{z}_{\text {tip }}$ pair of accelerometers and the TEO pair of control surfaces on the right and left wings. The block diagram for digital implementation ${ }^{2}$ of the symmetric and antisymmetric FSS control laws is shown in Figure 4. The accelerometer outputs from the left and right wing were passed through $25 \mathrm{~Hz}$ first-order antialiasing filters, modeled by the transfer function $157 /(s+157)$ and converted into digital data at a sampling rate of $200 \mathrm{~Hz}$. The digital controller separated the data into symmetric and antisymmetric components, computed the digital control law

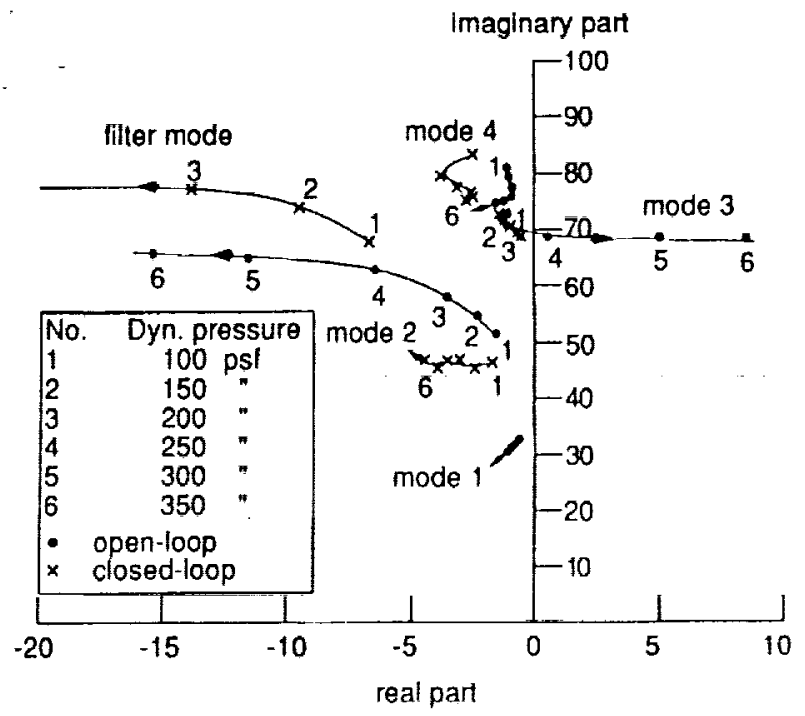

Figure 2. Symmetric open- and closed-loop dynamic pressure root-locus at $M=0.5$ (arrows indicate increasing dynamic pressure).

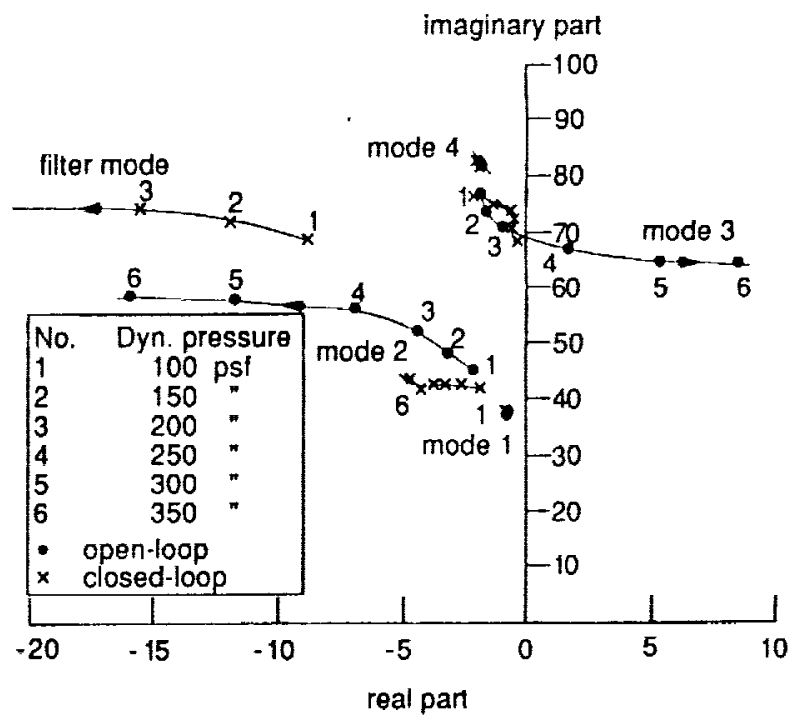

Figure 3. Antisymmetric fixed-in-roll open- and closed-loop dynamic pressure root-locus at $\mathrm{M}=0.5$ (arrows indicate increasing dynamic pressure).

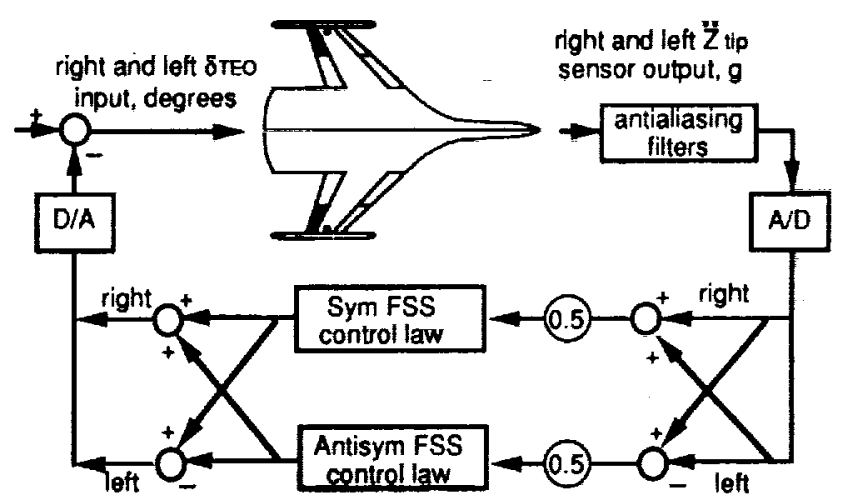

Figure 4. Digital FSS control law implementation block diagram. 
outputs and then distributed the processed feedback signals to the right and left actuators after 0.005 seconds computational delay.

Design plant model: The 68th order antisymmetric state-space equations at $q=350$ psf for the fixed-in-roll configuration was used as the design plant model, since from the analysis and the 1989 test, the antisymmetric flutter mode was found to be most critical and was encountered at a lower dynamic pressure, than the symmetric flutter mode. The accelerometer sensors and control surfaces were selected based on the frequency response analysis of the open-loop system. The Z̈TEO and żtip accelerometer responses were predominant at the wing-tip torsion frequencies due to the excitation from TEI and TEO control surfaces. In addition, the $\ddot{z}_{t i p}$ sensor exhibited relatively low response at frequencies above $25 \mathrm{~Hz}$. Therefore, $\ddot{z}_{\text {TEO and }}$ żtip accelerometer sensors and TEI and TEO control surfaces were initially studied as candidates for measurement inputs and control outputs, respectively.

Full order LQG design: A full order LQG control law was designed using the design plant model state-space equations (1) and (2). The full-order LQG control law which is given by equations (3) and (4), minimizes a weighted quadratic cost function defined by $E\left[y^{T} Q_{1} y+u^{T} Q_{2} u\right]$, where $Q_{1}$ and $Q_{2}$ are the plant output and control input weighting matrices 4,5 .

$$
\begin{aligned}
\mathrm{dx}_{\mathrm{d}} / \mathrm{dt} & =A_{\mathrm{o}} \mathrm{x}_{\mathrm{c}}+\mathrm{B}_{\mathrm{oy}}, \\
\mathrm{u} & =\mathrm{C}_{0} \mathrm{x}_{\mathrm{c}},
\end{aligned}
$$

where

$$
\begin{aligned}
& A_{0}=\left[F-B_{0} H+G C_{o}\right] \\
& B_{0}=P^{T} R_{v}{ }^{-1} \\
& C_{0}=-Q_{2}{ }^{-1} G^{T} S .
\end{aligned}
$$

The matrices $\mathrm{B}_{0}$ and $\mathrm{C}_{0}$ are the Kalman state estimator gains and the full-state optimal regulator gains, respectively. The matrices $P$ and $S$ are the positive definite solution of the steady state dual matrix Riccati equations, given by

$$
\begin{aligned}
& F P+P F T+G_{W} R_{w} G_{w}{ }^{T}-P H^{T} R_{v}{ }^{-1} H P=0 \\
& S F+F^{T} S+H^{T} Q_{1} H-S G \cdot Q_{2}^{-1} G^{T} S=0,
\end{aligned}
$$

where $R_{w}$ and $R_{v}$ denote the intensity matrices of the gust input and measurement Gaussian white noise processes, $w$ and $v$, respectively. To obtain the LQG control law, full-state optimal regulator gain matrix $C_{0}$ was first determined using a unit output weighting matrix, $\mathrm{Q}_{1}=\mathrm{I}$, and a control weighting matrix $\mathrm{O}_{2}=0.001 \mathrm{I}$, where $\mathrm{I}$ is a $2 \times 2$ identity matrix. Then the Kalman state estimator gain matrix $B_{0}$ was determined using $R_{w}$ $=0$ and $R_{V}=I$. The final selection of these weighting and noise intensity matrices for the full order control law, and the subsequent order reduction process were determined after several design iterations, until a stabilizing low order controller was found for the nominal design plant model. The control law order reduction process is described next.

Order Reduction: The full 68th order LQG control law given by equations (3) and (4) was first block-diagonalized, and then reduced to 11 th order by residualization of all the damped modes above $19 \mathrm{~Hz}$. Equations (3) and (4) in block-diagonalized form, are shown in equations (5) and (6), where the vector $x_{c l}$ represents the retained states and the vector $x_{\mathrm{c} 2}$ represents the remaining states associated with the damped higher frequency dynamics.

$$
\begin{aligned}
\frac{d}{d t}\left\{\begin{array}{l}
x_{c 1} \\
x_{c 2}
\end{array}\right\} & =\left[\begin{array}{cc}
A_{o 1} & 0 \\
0 & A_{02}
\end{array}\right]\left\{\begin{array}{l}
x_{c 1} \\
x_{c 2}
\end{array}\right\}+\left[\begin{array}{l}
B_{01} \\
B_{02}
\end{array}\right] y \\
u & =\left[\begin{array}{ll}
C_{01} & C_{02}
\end{array}\right]\left\{\begin{array}{l}
x_{c 1} \\
x_{c 2}
\end{array}\right\}
\end{aligned}
$$

In the residualization procedure, only the steady state part of the stable higher frequency dynamics in equation (5) were retained. This was accomplished by setting the state derivative $\mathrm{dx}_{\mathrm{c} 2} / \mathrm{dt}$ to zero and solving for $x_{c 2}$, provided the matrix $A_{02}$ is nonsingular (Ref. 4 ). The reduced state space model of the control law is given by equations (7) and (8).

where

$$
\begin{aligned}
\mathrm{d} x_{c} / \mathrm{dt} & =A x_{c}+B y \\
\mathrm{u} & =C x_{c}+D y
\end{aligned}
$$

$$
\begin{aligned}
x_{c} & =x_{c 1}, B=B_{01}, C=C_{o 1} \\
\text { and } D & =-C_{02} A_{02}-1 B_{02} .
\end{aligned}
$$

This procedure introduced a direct feedthrough matrix $D$ in equation (8). The residualized 11 th-order control law was subsequently reduced to a second-order control law by balanced realization and truncation of the balanced system. The balanced realization procedure finds a linear transformation in which the control law states have equal controllability and observability properties ${ }^{4}$. The weakly controllable and observable states are then truncated. Even with the elimination of these states, the resulting set of equations retained the most important input output characteristics of the original system. This second-order, two-input two-output control law, is given by equations (9) and (10).

$$
\begin{gathered}
\frac{\mathrm{dx}_{\mathrm{c}}}{\mathrm{dt}}=\left[\begin{array}{ll}
-5.2 & 64.6 \\
-64.6 & -5.2
\end{array}\right] \mathrm{x}_{\mathrm{c}}+\left[\begin{array}{cc}
1.25 & 1.95 \\
-0.45 & -0.73
\end{array}\right]\left[\begin{array}{l}
\ddot{z}_{\mathrm{TEO}} \\
\ddot{z}_{\text {tip }}
\end{array}\right\} \\
\left\{\begin{array}{l}
\delta_{\mathrm{TE}} \\
\delta_{\mathrm{TEO}}
\end{array}\right\}=\left[\begin{array}{cc}
-0.4 & 2.1 \\
3.6 & -9.4
\end{array}\right] \mathrm{x}_{\mathrm{c}}+\left[\begin{array}{cc}
-0.06 & -0.09 \\
0.13 & 0.21
\end{array}\right]\left[\begin{array}{l}
\ddot{z}_{\mathrm{TEO}} \\
\ddot{z}_{\text {tip }}
\end{array}\right\}
\end{gathered}
$$

The corresponding Bode diagrams of the four components of this $2 \times 2$ control law are shown in Figure 5 . This figure indicates that the maximum gain of this control law was $2.5 \mathrm{deg} / \mathrm{g}(8 \mathrm{~dB})$ with a peak gain at $10.3 \mathrm{~Hz}$. The primary stabilizing gain of this control law was from the sensor $\ddot{z}_{\text {ip }}$ to the control surface $\delta_{\text {TEO }}$. Although this control law stabilized the symmetric and antisymmetric plant models at $350 \mathrm{psf}$, the step responses contained high frequency components. With the addition of 25 $\mathrm{Hz}$ antialiasing filters to each accelerometer channel, the high frequency components of the step responses were eliminated. However, with the addition of $\mathrm{T}=0.005$ second computational delay ( modeled by the first-order Pade approximation $(2 / \mathrm{T}-\mathrm{s}) /(2 / \mathrm{T}+\mathrm{s}))$, the system was marginally stable. It was also noted that, when this control law was reduced to a single-input single-output (SISO) control law by retaining only the control law input $\bar{\varkappa}_{\text {tip }}$ and the output $\delta_{\text {TEO }}$ the nominal design plant was also stable. This simplified SISO control law was therefore, studied further in order to compensate for the computational delay effects, and possible uncertainty in the actual flutter frequencies, as mentioned earlier.

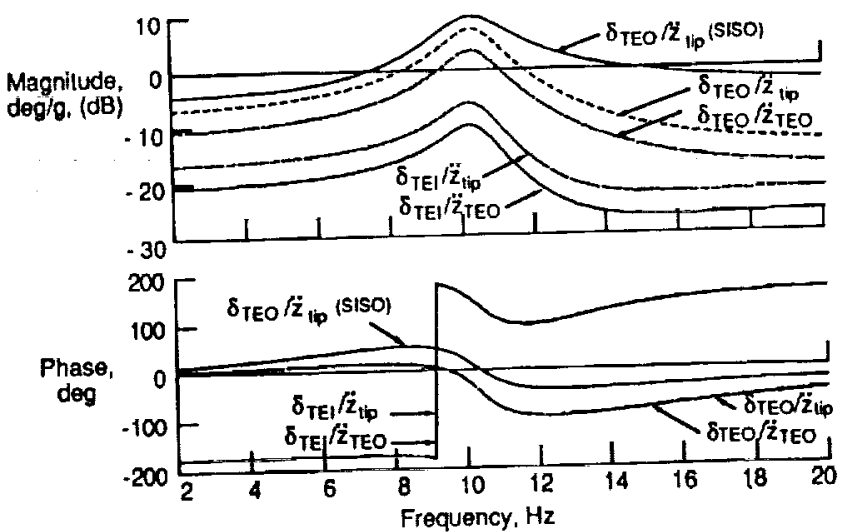

Figure 5. Bode diagram of reduced, second-order control laws . 


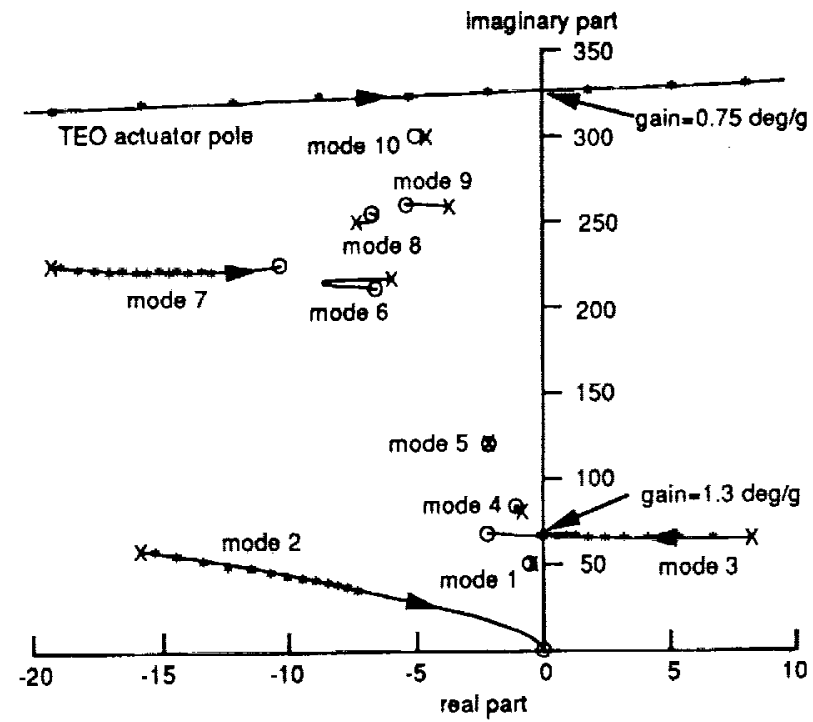

Figure 6. Gain root-locus plot for negative feedback from żtip to $\delta_{\text {TEO }}$ at $350 \mathrm{psf}$, antisymmetric fixed-in-roll configuration $(x=$ poles, $0=$ zeros, $*$ indicates gain increment by 0.1$)$.

SISO Control Law: This simplified SISO control law (plot

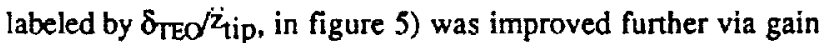
augmentation. The required gain level was determined using a gain root-locus analysis. The output gain feedback root-locus of the design plant model at $350 \mathrm{psf}$, with $\delta_{\text {TEO }}$ as plant input, and $\ddot{z}_{\text {tip }}$ as plant output, is shown in figure 6 . This root-locus indicated that, the open-loop unstable pole (mode 3) near $11 \mathrm{~Hz}$ migrated into the stable left half plane, with a negative feedback gain of $1.3 \mathrm{deg} / \mathrm{g}$ from $\ddot{z}_{\text {tip }}$ to $\delta_{\text {TEO }}$. However, the actuator poles near $50 \mathrm{~Hz}$ become unstable at a gain of $0.75 \mathrm{deg} / \mathrm{g}$. Therefore, a gain level of at least $1.3 \mathrm{deg} / \mathrm{g}$ in the 8 to $12 \mathrm{~Hz}$ frequency range, with subsequent gain attenuation at higher frequencies was necessary to stabilize the system, and accommodate the possible difference between the analytical and experimental flutter frequencies. In addition, compensation for the phase lag effects of the antialiasing filter and one cycle computational delay was also required. The total phase lag introduced by these two effects, was about 40 degrees at the frequency $10 \mathrm{~Hz}$.

The gain and phase compensations were achieved by varying the three elements of C and D in the SISO control law, and studying the gain and phase diagrams and the closed loop stability responses. An increase in $C_{1}$ and decrease in $\left|C_{2}\right|$ resulted in a desirable phase increase at low frequencies. An increase in D, reduced the phase (towards zero) at high frequencies, which was also beneficial. These three parameters were varied, until a gainlevel near $1.3 \mathrm{deg} / \mathrm{g}$ ( $2.3 \mathrm{~dB})$ was maintained over the frequency range 8 to $12 \mathrm{~Hz}$, and sufficient phase lead was obtained. The real part of the control law complex pole was also moved from -5.2 to -6.0 to achieve a wider gain range. The high frequency gain was kept below $0.75 \mathrm{deg} / \mathrm{g}$. This modified SISO control law is given by equations (11) and (12), assuming negative feedback.

$$
\begin{aligned}
& \frac{d x_{c}}{d t}=\left[\begin{array}{cc}
-6.0 & 64.6 \\
-64.6 & -6.0
\end{array}\right] x_{c}+\left[\begin{array}{c}
1.95 \\
-0.73
\end{array}\right] \ddot{z}_{t i p} \\
& \delta_{\text {TEO }}=\left[\begin{array}{ll}
14.4 & -3.1
\end{array}\right] x_{c}+0.63 \ddot{z}_{\text {tip }}
\end{aligned}
$$

The corresponding gain and phase plots are shown in figure 5 and are labeled $\delta_{\text {TEO }}{ }_{\text {żtip }}$ (SISO). The complex poles and zeros of this control law were $-6 \pm j 64.6$ and $-30 \pm j 56$, respectively. A second-order notch filter, given by the iransfer function $\left(s^{2}+42 s+44100\right) /\left(s^{2}+84+44100\right)$, was added to increase the symmetric model gain margin to $6 \mathrm{~dB}$, near $33 \mathrm{~Hz}$. This filter attenuated a $33 \mathrm{~Hz}$ lightly damped oscillation due to the interaction of the sixth and seventh symmetric flexible modes. $A$ first-order washout filter, given by the transfer function $s /(s+6)$ was also added to remove any steady state input bias to the sensor signal.

Discretization: The resulting 5th order SISO control law in Laplace domain was discretized using the Tustin transformation $\mathrm{z}=(\mathrm{I}+\mathrm{sT} / 2) /(1-\mathrm{sT} / 2)$, where $\mathrm{T}$ is the sampling interval. For the $200 \mathrm{~Hz}$ sampling rate used by the digital controller, $\mathrm{T}=0.005$ seconds. With the Tustin transformation at this sampling rate, the Bode diagrams in the Laplace domain and the discrete domain were almost identical below $15 \mathrm{~Hz}$. Hence no frequency warping corrections were applied.

Dynamic-pressure root-locus: The open- and closed-loop dynamic pressure root-locus plots are compared in figures 2 and 3 . These comparisons indicated that both the symmetric and antisymmetric models were stable, up to dynamic pressure $q=$ 350 psf. The closed-loop frequency decoupling was due to lowering of the frequency of mode 2 to about $6.8 \mathrm{~Hz}$. The frequency of mode 3 was increased to $11.6 \mathrm{~Hz}$, but the damping ratio was only of the order 0.010 at 300 psf.

Sensitivity studies: The closed-loop system sensitivity was studied by perturbing the second and third modal frequencies in the state-space block-diagonalized plant model by $\pm 10 \%$ and the nominal gains by $\pm 4 \mathrm{~dB}$ at $\mathrm{q}=250 \mathrm{psf}$ and examining the closed-loop system step responses, for all possible combinations. These studies indicated that the design could accommodate simultaneous gain and frequency changes for all cases except when the second and third mode frequencies were perturbed to approach each other. Sensitivity studies were also done using the state-space model with and without the $25 \mathrm{~Hz}$ antialiasing filters, with and without one cycle delay, with additional delays, and with $\pm 6 \mathrm{~dB}$ gain perturbations at $250 \mathrm{psf}$. These studies indicated that the symmetric configuration could tolerate one additional delay (or phase lag of $1.8 \mathrm{degrees} / \mathrm{Hz}$ ) at half the nominal gain, but the antisymmetric configuration would become unstable with an $11 \mathrm{~Hz}$ oscillation. The phase and gain margin comparisons with the experimental results, described in the next section, indicated that this particular situation may have been encountered during the experiment. The gain loss was apparent from the experimental Bode diagram.

\section{Summary of Test Results}

Open-loop Flutter: Based on examination of the peak-hold data obtained during the wind tunnel test with the tip ballast store coupled, the open-loop $(O L)$ flutter dynamic pressures were as follows: The free-to-roll $O L$ symmetric flutter was at a dynamic pressure of $235 \mathrm{psf}$, at a frequency of $9.6 \mathrm{~Hz}$. The fixed-in-roll $O L$ antisymmetric flutter was at a dynamic pressure of $219 \mathrm{psf}$, at a frequency of $9.1 \mathrm{~Hz}$. These experimental symmetric and antisymmetric $O L$ flutter dynamic pressures were, respectively, 13 and 14 psf below the predicted values, and the flutter frequencies were, respectively, $1.6 \mathrm{~Hz}$ and $1.8 \mathrm{~Hz}$ below the predicted values.

Qpen-loop frequency responses: Figures 7 and 8 show the $O L$ frequency responses of $\ddot{z}_{\text {tip }}$ due to $\delta_{\text {TEO }}$ from analysis and experiment at $250 \mathrm{psf}$, for the symmetric and antisymmetric (fixed-in-roll) cases, respectively. At this dynamic pressure, the $O L$ plant is unstable. So, the $O L$ frequency responses were computed from closed-loop $(C L)$ experimental data, using the Controller Performance Evaluation (CPE6,7) procedure. Figure 7 indicates good agreement below $9 \mathrm{~Hz}$ and qualitative agreement above $12 \mathrm{~Hz}$. Above $12 \mathrm{~Hz}$, the magnitudes differ by about $5 \mathrm{~dB}$ while the phase angles are nearly equal. Figure 8 indicates fair agreement, below $7 \mathrm{~Hz}$, and qualitative agreement 

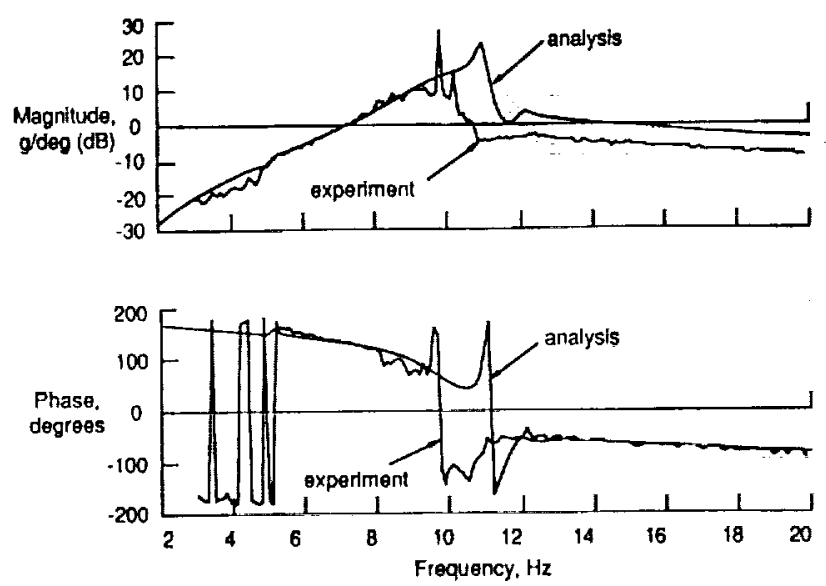

Figure 7. Comparison of $\ddot{z}_{\mathrm{ip}} / \delta_{\text {TEO }}$ Bode diagrams at $250 \mathrm{psf}$, symmetric configuration.
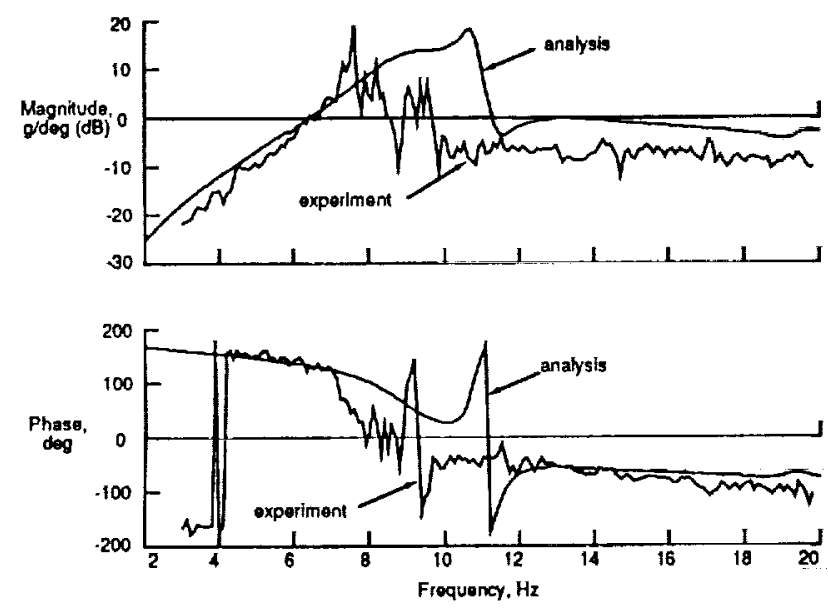

Figure 8. Comparison of $\ddot{z}_{\text {tip }} / \delta_{\text {TEO }}$ Bode diagram at $250 \mathrm{psf}$, antisymmetric fixed-in-roll configuration.

above $12 \mathrm{~Hz}$. Above $12 \mathrm{~Hz}$, the magnitudes differ by 6 to $8 \mathrm{~dB}$ and the phase angles differ by 10 to 20 degrees. Note, that for each phase diagram, the 180 degree crossing occurs near the respective $O L$ flutter frequencies, and the difference between their predicted and experimental values is quite apparent.

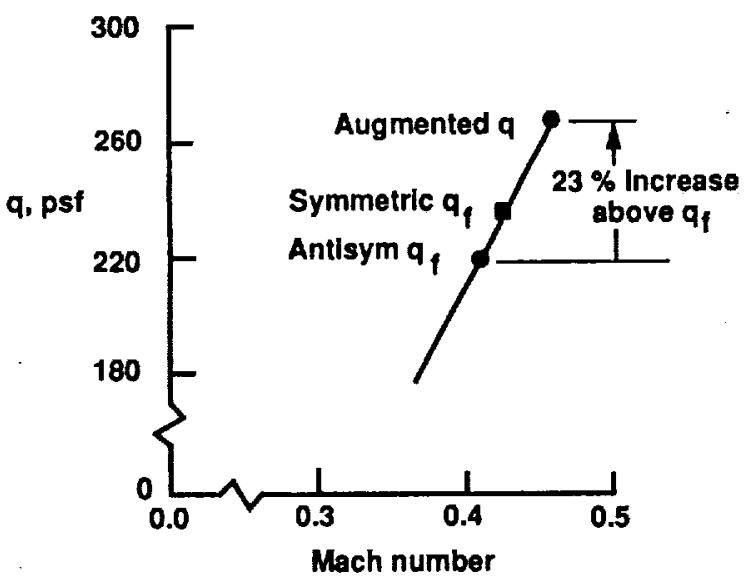

Figure 9. Summary of results for fixed-in-roll FSS wind-tunnel test.
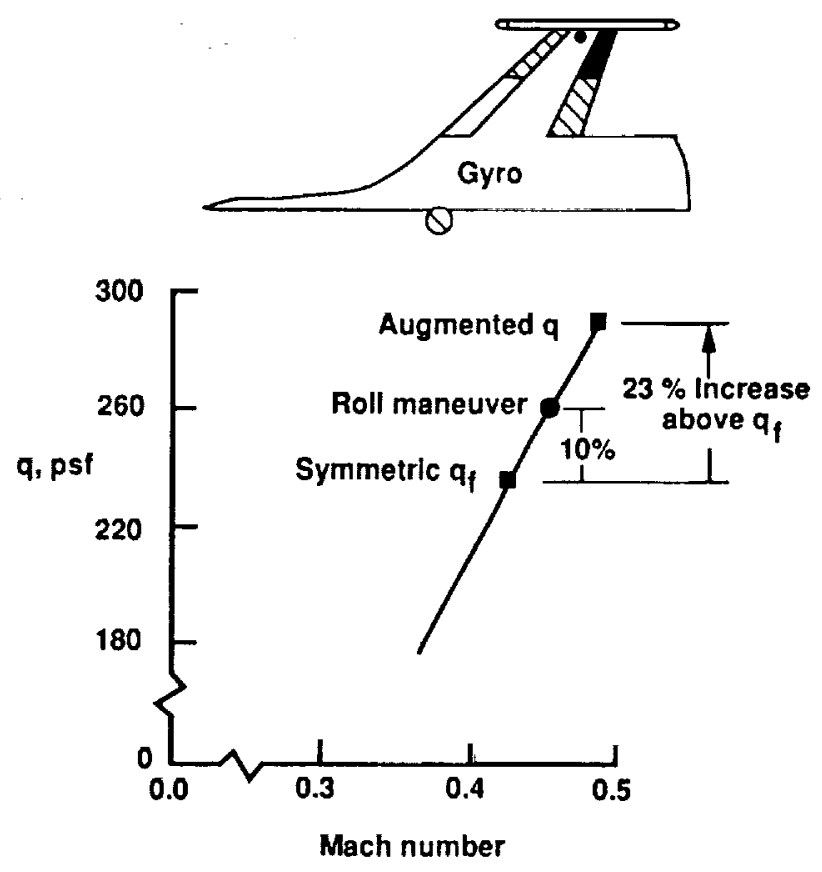

Figure 10. Summary of results for free-to-roll RMLA/FSS wind-tunnel test.

Closed-loop Tests: The active flutter suppression test results are summarized in figures 9 through 13 . Figures 9 and 10 show the wind-tunnel test dynamic pressures versus the free stream Mach number. During the wind-tunnel test, in the fixed-in-roll configuration, with both the symmetric and antisymmetric FSS control laws operating, the $C L$ system was stable up to $q=270$ psf, at Mach 0.46 . This augmented q represents a $23 \%$ increase over the $O L$ antisymmetric qf.

During the wind-tunnel test, in the free-to-roll configuration, with the symmetric FSS control law operating, the $C L$ system was stable up to $q=290 \mathrm{psf}$, at Mach 0.48. This augmented $q$ represents a $23 \%$ increase over the $O L$ symmetric of as shown in figure 10. This FSS control law also suppressed the flutter when a Rolling Maneuver Load Alleviation (RMLA ${ }^{8}$ ) system was tested with rapid roll maneuvers at $q=260 \mathrm{psf}, 11 \%$ above the $O L$ symmetric flutter boundary. This RMLA control law used LEO and TEI control surfaces, so the interaction with the FSS control law was minimal.

The rms deflection and deflection rate of the right and left side TEO control surface were computed from the data sampled at $200 \mathrm{~Hz}$ at each fixed-in-roll FSS test condition. If the value of the right and left differed, the maximum is plotted in figure 11 . The maximum rms deflection and rates were less than 0.4 degrees and 25 degrees/second, respectively. These maximum rms deflection and rate demands of the actuators were well below the maximum allowable values of $1 \mathrm{deg}$ and $75 \mathrm{deg} / \mathrm{sec}$ as stated earlier in the paper.

The Nyquist-diagram-based gain- and phase-margins were estimated using the CPE technique, during the experiment. These estimates were compared with corresponding analytical quantities in figures 12 and 13 , for the symmetric free-to-roll and the antisymmetric fixed-in-roll configurations, respectively. For the symmetric, free-to-roll configuration (figure 12), the 


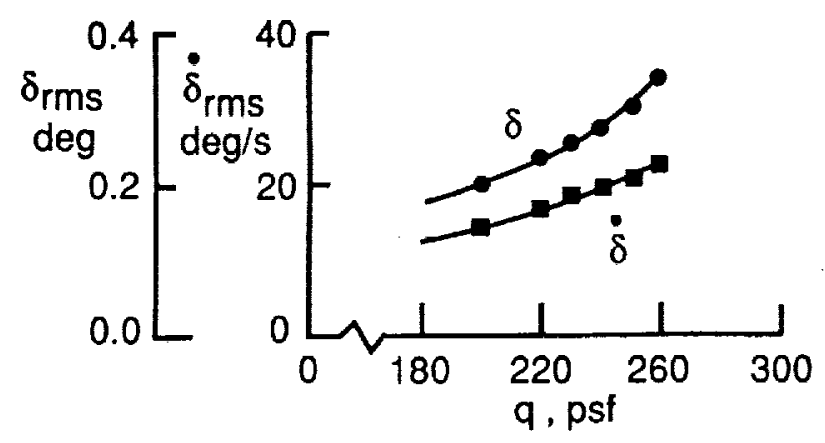

Figure 11. Maximum $\delta_{\text {TEO }}$ control surface deflection and rates demands for simultaneous symmetric and antisymmetric flutter suppression tests.

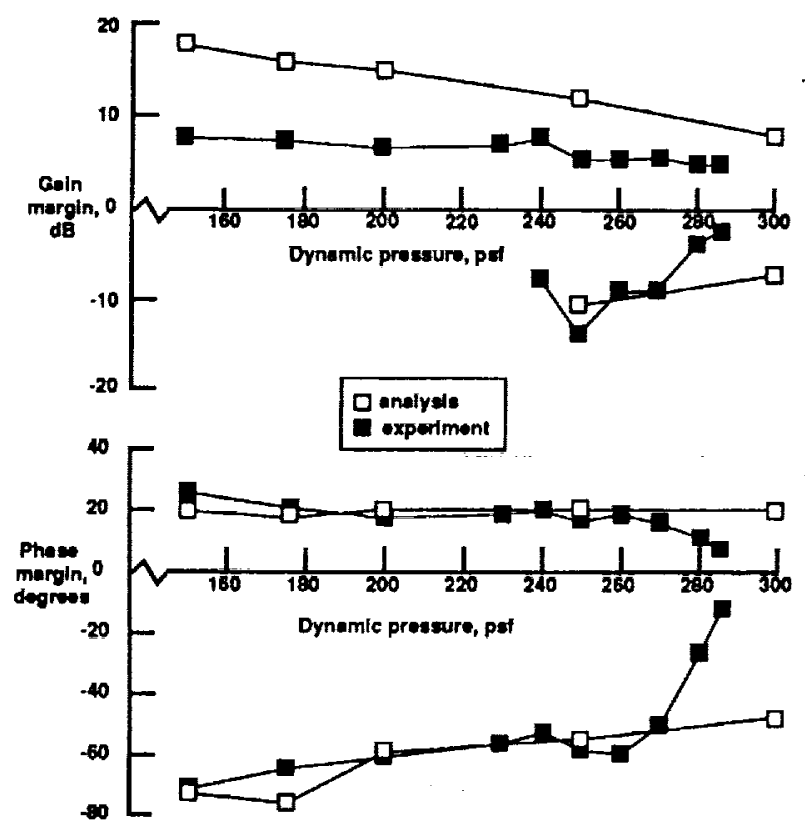

Figure 12. Gain and phase margin comparison (symmetric).

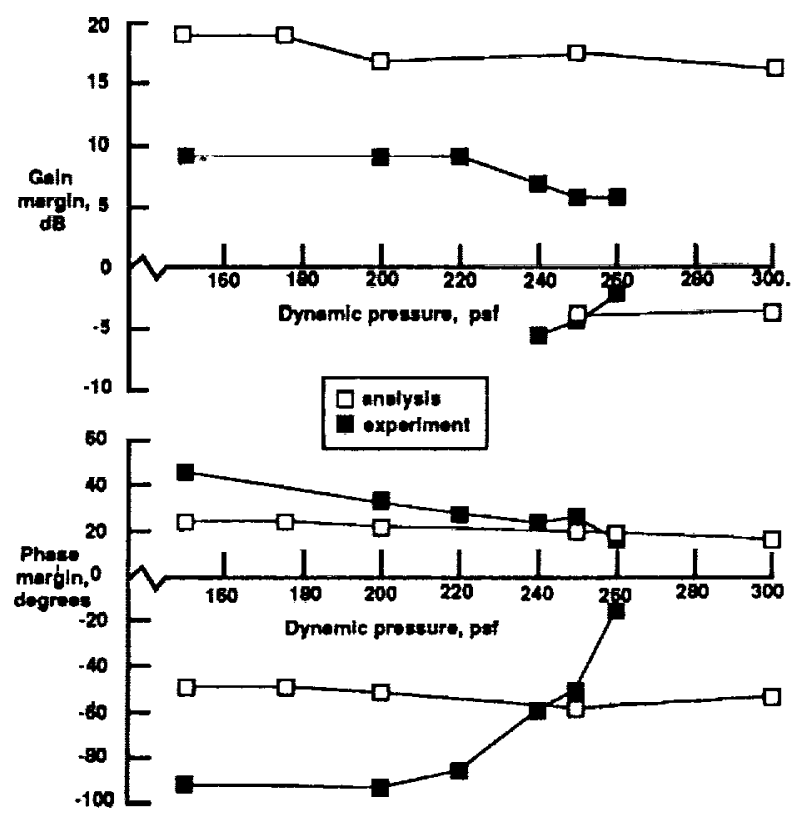

Figure 13. Gain and phase margin comparison (antisymmetric, fixed-in-roll). analytical and experimental gain margins were above $\pm 6 \mathrm{~dB}$ up to $270 \mathrm{psf}$. The analytical positive phase margins (at or below 7 $\mathrm{Hz}$ ) were about 20 degrees, but the negative phase margins (at or above $12 \mathrm{~Hz}$ ) were well above 45 degrees. The analytical phase margins were close to experimental results up to about 270 psf.

For the antisymmetric, fixed-in-roll configuration (figure 13), the analytical negative gain margins were only $-3 \mathrm{~dB}$. The analytical positive phase margins (at or below $7 \mathrm{~Hz}$ ) were about 20 degrees, but the negative phase margins (at or above $12 \mathrm{~Hz}$ ) were 45 degrees. The analytical phase margins were close to the experimental data at $250 \mathrm{psf}$, because the design model was fairly accurate at frequencies below $7 \mathrm{~Hz}$ (see figure 8). The negative gain and phase margins at the high frequency end were primarily responsible for preserving the system stability. The sdurce of additional phase lag with increasing dynamic pressure was possibly due to highly loaded actuators. The gain loss was apparent from the experimental Bode diagram shown in figure 8 in the 8 to $12 \mathrm{~Hz}$ frequency range.

\section{v. Conclusions}

A single-input single-output control law was designed for flutter suppression using linear quadratic Gaussian theory and involved control law order reduction, a gain root-locus study and use of previous experimental results. The control law was digitally implemented and tested. Simultaneous suppression of symmetric and antisymmetric flutter modes in close proximity was demonstrated to $23 \%$ above the open-loop antisymmetric flutter boundary when the model was in a fixed-in-roll configuration. Symmetric flutter suppression system operating simultaneously with a rolling maneuver load alleviation system was tested to $23 \%$ above the open-loop symmetric flutter boundary, when the model was in a free-to-roll configuration. With this combined system, rapid roll maneuvers were also performed at $11 \%$ above the symmetric flutter boundary.

\section{References}

1 Perry, B. III, Cole, S. R and Miller, G. D., "A Summary of the Active Flexible Wing Program," AIAA Paper 92-2080, presented at the 1992 Dynamics Specialists Meeting, April 16$17,1992$.

2 Perry, B. III, Mukhopadhyay, V., Hoadley, S. T., Cole, S. R., Buttrill, C. S. and Houck, J. A., "Digital Implementation, Simulation and Testing of Flutter-Suppression Systems for the Active Flexible Wing Wind-Tunnel Model," AIAA Paper 901074, Proc. of 31st AIAA SDM Conference, Long Beach, CA, April 1990.

${ }^{3}$ Buttrill, C. S., Bacon, B. J., Heeg, J. and Houck, J. A., "Simulation and Model Reduction for the AFW Program," AIAA Paper 92-2081, presented at the 1992 Dynamics Specialists Meeting, April 16-17, 1992.

${ }^{4}$ Maciejowski, J. M., Multivariable Feedback Design, Addison Wesley Publishing Co., Great Britain, 1989.

5 Bryson, A. E., Jr, and Ho, Y. C., Applied Optimal Control, Hemisphere Publishing Corporation, Washington, 1975.

6 Pototzky, A. S., Wieseman, C. D., Hoadley, S. T. and Mukhopadhyay, V., "Development and Testing of Methodology for Evaluating the of Performance of Multiinput/multi-output Digital Control Systems," AIAA Paper 90 3501, presented at the AIAA Guidance, Navigation and Control Conference, Portland, Oregon, August 1990.

7 Pototzky, A. S., Wieseman, C. D., Hoadley, S. T. and Mukhopadhyay, V., "On-line Performance of Multi-loop Digital Control Systems", Journal of Guidance, Control, and Dynamics, Vol. 15, No. 3, May-June, 1992 (TBP).

8 Woods-Vedeler, J. A. and Pototzky, A. S., "Rolling Maneuver Load Alleviation Using Active Controls," ALAA Paper 92-2099, presented at the 1992 Dynamics Specialists Meeting, April 16-17, 1992. 


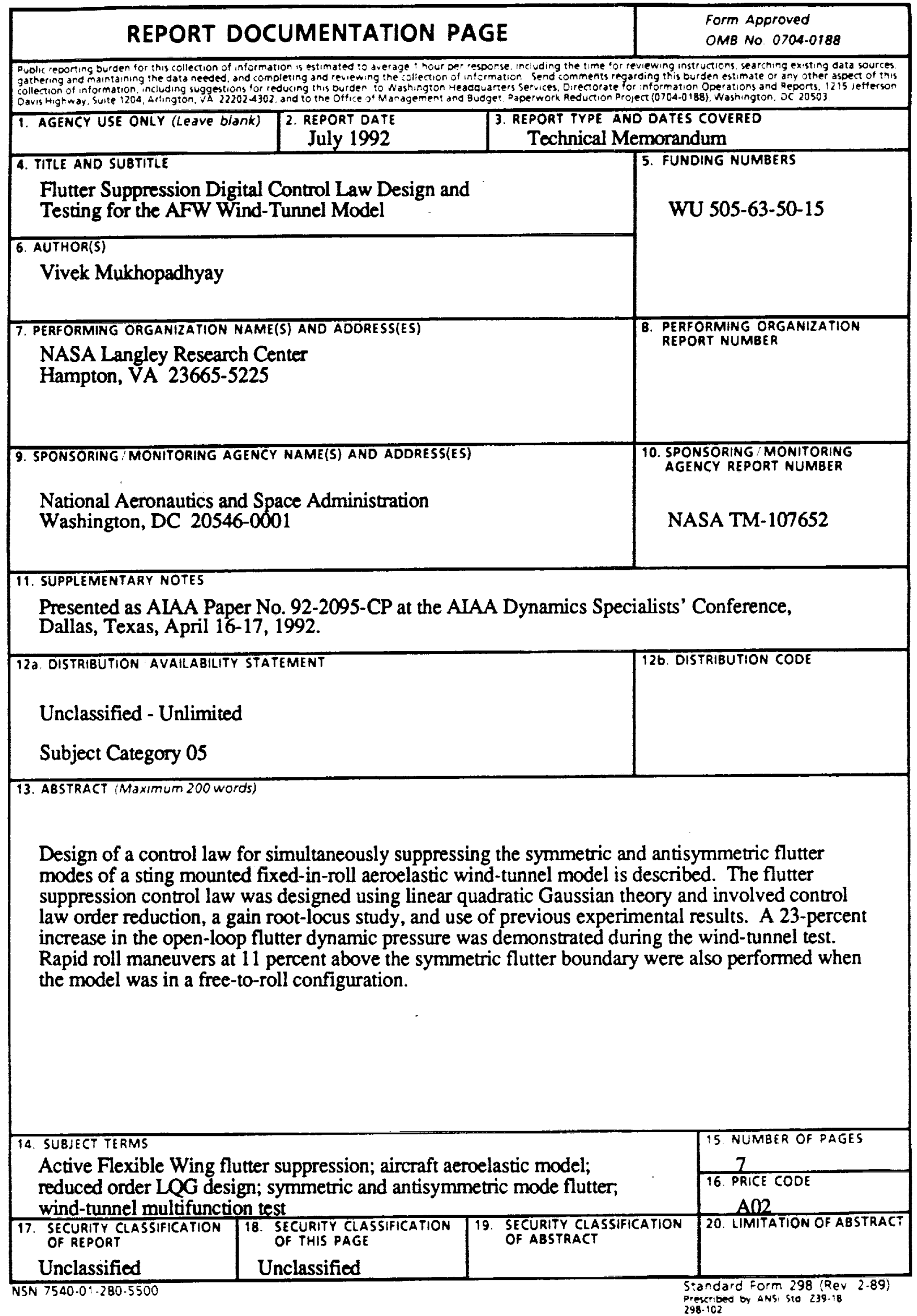

